\title{
Assessment of Patient and Caregiver Experiences of Dementia-Related Symptoms: Development of the Multidimensional Assessment of Neurodegenerative Symptoms Questionnaire
}

\author{
Dona E.C. Locke ${ }^{a}$ Kara B. Dassel ${ }^{c}$ Geri Hall $^{d}$ Leslie C. Baxter ${ }^{c}$ \\ Bryan K. Woodruffa Charlene Hoffman Snyder ${ }^{\mathrm{a}}$ Bruce L. Miller \\ Richard J. Casellib

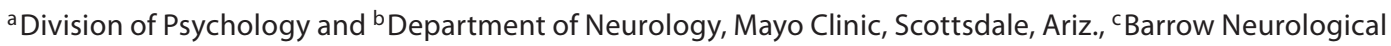 \\ Institute, and ${ }^{d}$ Banner Alzheimer's Institute, Phoenix, Ariz., and ${ }^{~}$ Department of Neurology and Memory and \\ Aging Center, University of California, San Francisco, Calif., USA
}

\section{Key Words}

Multidimensional questionnaire $\cdot$ Neurodegenerative

disorders $\cdot$ Validation

\begin{abstract}
Background: To provide preliminary validation data on a self- or informant-report multidimensional questionnaire of symptoms associated with neurodegenerative disorders. Methods: Participants from 2 trials $(n=125)$, the Arizona APOE Cohort and the Arizona Alzheimer's Disease Center, completed the Multidimensional Assessment of Neurodegenerative Symptoms questionnaire (MANS) and other related measures. Results: Measures of central tendency are provided for the sample as a whole and by cognitive status. Internal consistency of the MANS is excellent ( $\alpha=0.98$ ). Factor analysis suggests 4 factors. Correlational analyses support the construct validity of the MANS with moderate to high ( $r=0.54-0.87$ ) correlations between the MANS and measures of mood, cognition and daily functioning. Conclusion: Results provide initial support for the MANS as a brief measure that is a reliable and valid indicator of cognitive,
\end{abstract}

personality, functional and motor symptoms potentially related to neurodegenerative etiologies. Further research with the MANS is warranted.

Copyright $\odot 2009$ S. Karger AG, Basel

Neurodegenerative disorders such as Alzheimer's disease (AD), Lewy body disease and frontotemporal dementia (FTD) are becoming more prevalent as the population ages, resulting in significant caregiving and financial burdens. For example, in the United States AD is the most common neurodegenerative illness, with an estimated 5.2 million Americans currently diagnosed. It is projected that by the year 20307.7 million people will be affected and by 2050 11-16 million people will be affected if there are no changes in treatment options. The direct financial costs to Medicare and Medicaid for care for people with $\mathrm{AD}$ and the indirect costs to businesses for employees who are caregivers are estimated to be over USD 148 billion per year. This estimate does not include the costs paid for by the Department of Veteran's Affairs, private health insurance, long-term care insur-

Dona E.C. Locke, $\mathrm{PhD}$

Division of Psychology, Mayo Clinic

13400 E. Shea Boulevard

Scottsdale, AZ 85259 (USA)

Tel. +1 480301 8297, Fax +1 480301 6258, E-Mail locke.dona@mayo.edu 
ance or out-of-pocket expenses for patients and their families [1]. Mild cognitive impairment (MCI) [2] is also a growing concern among clinicians since persons with MCI are at increased risk for progression to dementia (6-25\% per year) compared to the general population $(1-2 \%)[2,3]$.

In conjunction with investigation of therapeutic treatments and disease modifying agents, it is important to increase our ability to detect neurodegenerative disorders early in the disease course. This early detection and diagnosis is imperative to the preservation of cognitive and physical functioning through therapeutic interventions, which will, in turn, minimize financial costs and emotional burden to individuals, family members and society at large. Thus, in addition to continued research into developing effective treatment options, an important parallel line of research is early detection of change so any new effective treatment can be implemented at a time to maintain maximal functional status.

Neurodegenerative disorders are generally characterized by subjective cognitive complaints, objective evidence of impairment on neuropsychological tests, changes in personality and/or mood, and difficulty in performance of activities of daily living [4]. In order to properly assess and diagnose underlying neurodegenerative diseases, one important component of the evaluation is gathering detailed and accurate information from both the patient and an informant about the patient's symptoms in multiple domains. In addition to subjective reports of patient symptomology, patients undergo a thorough medical evaluation, which typically involves a review of the patient's medical history, physical and neurological exam, neuropsychological evaluation, neuroimaging and blood work. However, the patient's or caregiver's report is often the impetus for a diagnostic work-up, which stresses the importance of gathering selfreport data across several symptom domains in a standard fashion.

Currently, there is a need for the development of a reliable and valid questionnaire specifically for older adults with suspected neurodegenerative diseases that can be used in clinical settings. Self [5] and informant $[6,7]$ reports have been found to correlate with performance on cognitive tests, daily functioning and neurophysiologic changes. Furthermore, discrepancy between self and informant ratings may also be of diagnostic utility since patients with cognitive impairments tend to overestimate their abilities compared to ratings provided by informants [8]. Despite the potential usefulness, many of the questionnaires currently available do not have self and informant formats, thereby limiting comparability of results and potential diagnostic utility.

Second, many questionnaires are restricted in their scope in that they are single-dimensional (e.g. cognitive symptoms) as opposed to multidimensional (e.g. cognitive symptoms, motor symptoms, psychiatric symptoms), which is imperative in differential diagnosis of neurodegenerative disorders [9]. For example, the Neuropsychiatric Inventory [10], Activities of Daily Living (ADL) scales [11] and the Memory Functioning Questionnaire [12] assess single symptomatic domains of dementia including behavioral, physical and cognitive changes, respectively. Global assessment tools such as the Clinical Dementia Rating (CDR) scale [13] and the Global Deterioration Scale [14] are multi-dimensional, but are primarily used as staging instruments that emphasize cognitive and physical functioning.

Third, many of the existing questionnaires provide summary scores, or the sum of various functional domains. The summary score provides a general description of the patient's functioning, but does not give detailed information regarding the specific domains that may be affected and as a result may mask functional change [9]. Fourth, many questionnaires used coarse ratings scales such as 'independent', 'needs assistance' or 'dependent', which are not sensitive to subtle changes in functioning. Fifth, many current questionnaires were not developed for use within clinical settings and were developed and validated using diverse populations (e.g. psychiatric patients, younger adults, persons with specific health conditions such as arthritis, diabetes, or heart disease) and settings (e.g. community, assisted living facilities or nursing homes), which limits the sensitivity and specificity of the instrument when used within a clinical setting [15]. Lastly, many questionnaires are gender-biased and may focus on Instrumental Activities of Daily Living (IADL) items such as shopping, doing laundry, housekeeping chores or cooking, thus masking potential deficits in other complex gender-appropriate domains such as household maintenance and financial management [16].

Therefore, the goal of this study is to provide initial validation information on a novel questionnaire, the Multidimensional Assessment of Neurodegenerative Symptoms (MANS), which was designed to address the major limitations of extant measures. Specifically, the MANS is a multidimensional (i.e. ADL, cognitive, behavior and motor functioning) informant- and patient-based questionnaire, developed in a population of older adults with various levels of cognitive abilities, that assesses sub- 
tle changes over time in a gender-neutral manner, that provides global and domain-specific information and which may aid clinicians in making a differential neurodegenerative diagnosis.

\section{Methods}

\section{Participants}

Participants for this project were recruited from the Arizona APOE Cohort or the Arizona Alzheimer's Disease Center (ADC) Clinical Core, both of which are described in more detail below. Although there was some overlap in ages, most members of the Arizona APOE Cohort were under 70 years of age, with persons as young at 21 enrolled in the project. In order to restrict the age range to those persons at risk of dementia, we selected for this analysis consecutive participants from the APOE cohort who were age 60 and older. Alternatively, most members of the Arizona ADC Clinical Core were 70 years of age and older, with a range from 50-85. Inclusion and exclusion criteria were otherwise identical for these 2 cohorts. All individuals gave their written informed consent, and the study was approved by the institutional review board at Mayo Clinic Arizona.

A total of 125 participant and informant dyads completed the MANS as part of the APOE cohort $(n=47)$ or the ADC Clinical Core $(n=78) .78 \%$ of the informants were the spouse or live-in partner of the participant. The remaining informants were adult children of the participant (13.5\%), long-time friends of the participant $(6 \%)$ or siblings of the participant $(2.5 \%)$. These informants generally knew the participants quite well, as $96.6 \%$ of the informants had known the participant for at least 10 years or more. The remaining informants knew the participants for 7-9 years (1.4\%), $4-6$ years (1\%) and $1-3$ years (1\%).

The mean age of the participant sample was 70.5 years $(\mathrm{SD}=$ 7.4) with a range of 50-85. The sample was nearly evenly split along gender distribution with $54 \%$ of the participants being female. Broadly defined, 65 (52\%) of the participants were cognitively unimpaired and 60 (48\%) of the individuals had impaired cognition. Within the impaired group, diagnoses included possible and probable AD $(\mathrm{n}=18)$, MCI $(\mathrm{n}=16)$, FTD $(\mathrm{n}=10)$, probable Lewy body dementia $(n=3)$, posterior cortical atrophy $(n=$ $3)$, corticobasal degeneration $(n=2)$, progressive nonfluent aphasia $(n=2)$, progressive supranuclear palsy $(n=2)$, mixed etiology $(n=2)$, Parkinson's disease with dementia $(n=1)$ and dementia not otherwise specified $(\mathrm{n}=1)$.

\section{Arizona APOE Cohort}

Beginning in 1994, those recruited to the Arizona APOE Cohort $[17,18]$ were recruited through local newspaper advertisements that requested cognitively normal individuals who had a first degree relative with dementia. All potential participants received APOE genotyping. From the APOE test results, all of the APOE e4 homozygotes were evaluated along with 1 APOE e4 heterozygote and 2 APOE e4 noncarriers who were individually matched to each homozygote for their age (within 3 years), gender and educational level (within 2 years). Each prospective APOE Cohort recruit was then invited to return for the following screening tests: medical history, neurologic examination, Folstein MiniMental Status Exam (MMSE [19]), Hamilton Depression Rating
Scale (Ham-D [20, 21]), Structured Psychiatric Interview for DSM-III-R [22], Functional Assessment Questionnaire (FAQ, [23]) and IADL [11].

Participants with potentially confounding medical, neurological or psychiatric problems (such as prior stroke, traumatic brain injury, memory or other form of cognitive impairment, parkinsonism, major depression or substance abuse) were excluded. None of the participants in the APOE cohort met the published criteria for age-associated memory impairment [24], MCI [2, 3], $\mathrm{AD}$ [25] or any other form of dementia [22] or major depressive disorder [22] at the time of enrollment.

Those fulfilling these requirements were administered an extensive neuropsychological battery that was repeated every 2 years. Diagnostic status at entry and follow-up was determined by a behavioral neurologist and a psychiatrist as part of a longstanding collaborative study [18]. Beginning in 2007, all participants in the APOE cohort were also administered the CDR [13]. From that point forward, consecutive participants over the age of 60 were included in this analysis $(\mathrm{n}=47)$. One participant from the APOE cohort converted to probable $\mathrm{AD}$ at the evaluation included in this analysis, and is categorized as such, rather than as a normal control, in all subsequent analyses. The remaining 46 subjects were cognitively normal. As part of that extensive longitudinal follow-up, subjects and informants completed the self-report and informant-report MANS.

\section{Arizona Alzheimer's Disease Center Clinical Core}

Beginning in 2000, the ADC Clinical Core began recruiting and enrolling subjects through 5 participating institutions: Barrow Neurological Institute, Mayo Clinic Arizona, Sun Health Research Institute, the University of Arizona Health Sciences Center and the Tucson Veterans Administration Hospital. Data for the current study were obtained only from participants followed at the Mayo Clinic Arizona as this is the only ADC site that asks participants to complete the self-report and informant-report MANS at each visit $(n=78)$. Diagnostic status was determined initially by the neurologist enrolling the participant, but reviewed and agreed upon by consensus at a Diagnostic Clinical Core Conference comprised of member neurologists and neuropsychologists from each participating institution. Of the 78 ADC participants from Mayo, 19 were cognitively normal. The remaining participants had a variety of cognitive disorder diagnoses including possible and probable $\operatorname{AD}(n=17), \operatorname{MCI}(n=16), \operatorname{FTD}(n=10)$, probable Lewy body disease $(n=3)$, posterior cortical atrophy $(n=3)$, cortical-basal degeneration $(n=2)$, progressive nonfluent aphasia $(n=2)$, progressive supranuclear palsy $(n=2)$, mixed etiology $(n=2)$, Parkinson's disease with dementia $(n=1)$ and dementia not otherwise specified $(n=1)$.

\section{Measures}

As described in the introduction, we aimed to develop a questionnaire that captures self-report and informant-report of a variety of symptoms related to various neurodegenerative syndromes. The origin of this questionnaire stems from a telephone screening measure for FTD developed by an FTD working group of California State Alzheimer's Centers members. With their permission, our group reduced the number of items based on expert opinion of the clinical utility of the items, and then included additional items designed to capture symptoms that may be related to neurodegenerative etiologies other than FTD. We also added a
Locke/Dassel/Hall/Baxter/Woodruff/ Hoffman Snyder/Miller/Caselli 
semiquantitative scaling for rating the frequency of the symptoms of interest from 0 (never) to 4 (routinely), with intermediate values of 1 (once), 2 (occasionally) and 3 (more than monthly). We also adapted an informant version with identical questions.

The MANS is comprised of 87 questions that assess changes in daily habits, personality and motor functioning. It is written at a 7 th grade reading level and requires approximately 5-10 min to complete. The questionnaire is designed such that if a participant or informant reports that there has been no change within the broad domain, he/she can skip the individual items (which are assumed to remain unchanged) and move onto the next section of questions. Thus, each section begins with a yes/no question asking about presence/absence of change in daily habits, personality or movement in the past year. If the responder indicates there has been a change, he/she is asked to rate the frequency of that behavior on the $0-4$ scale described above. Because participants are typically evaluated on an annual basis in both clinical and research settings, the MANS instructs participants and informants to rate change and frequency of a behavior over the past year. The range of possible scores ranges from 0-348 with higher scores indicating more frequent and severe symptoms.

As part of the ADC and APOE cohort studies, participants complete or are administered a number of other measures that have some construct overlap with the MANS. The person administering and scoring the additional measures and questionnaires was blinded to participant and informant responses on the MANS at the time of administration or scoring. In the longitudinal battery, there are no other multidimensional self-report measures to provide direct concurrent validity of the MANS compared to a measure of all the same constructs. Thus, we provide support for the validity of the MANS utilizing similar, though not completely overlapping, construct measures that are commonly used in dementia-related research and clinical practice. These validation measures include: measures of mood (Ham-D [21], Geriatric Depression Scale [26]), a measure of behavioral symptoms (Neuropsychiatric Inventory [10]), measures of functional status (FAQ [23], CDR [13]), measures of global cognitive functioning (MMSE [19], Dementia Rating Scale-2, DRS-2 [27]) and a measure of motor functioning (Unified Parkinson Disease Rating Scale, UPDRS [28]). Small to moderate positive (Ham-D, Geriatric Depression Scale, Neuropsychiatric Inventory, FAQ, CDR, UPDRS) or negative (MMSE, DRS-2) correlations were considered supportive depending on the theoretical relationship between construct measures. Correlations $<0.3$ were considered small, those between $0.3-0.5$ were considered moderate and those $>0.5$ were considered large [29].

\section{Results}

\section{Internal Consistency}

Reliability analysis shows excellent internal consistency for both the self-report $(\alpha=0.98)$ and informant-report $(\alpha=0.98)$ versions of the MANS.

\section{Factor Analysis}

As described above, the MANS was conceptually designed with 3 broad categories of symptoms: changes in daily habits, changes in personality, and changes in motor skills. To better understand the psychometric factor structure, exploratory factor analysis using promax rotation was performed for both the self-report MANS and the informant-report MANS. A review of the responses and range of each individual question revealed one 'Have you had a convulsion?' - with no variance (e.g. all responders rated a 0 ). Therefore, this item was not included in the factor analysis. Based on the location of the 'elbow' of the scree plot, the percent of variance explained, as well as consistency across the 2 versions of the MANS, it was concluded that both versions of the MANS show 1 large factor as well as 3 additional smaller factors. Factor loadings of 0.4 or greater were utilized for individual item loadings. See tables 1 and 2 for individual item loadings for the self-report and informant-report MANS.

For the self-report MANS, the 4 factor structure explained $76.4 \%$ of the variance. Factor 1 explained $54.7 \%$ of the variance (eigenvalue $=31.1$ ), factor 2 explained an additional $8.7 \%$ of the variance (eigenvalue $=4.96$ ), factor 3 explained an additional $6.8 \%$ of the variance (eigenvalue $=3.86)$ and factor 4 explained $6.2 \%$ of the variance (eigenvalue $=3.51$ ). A review of the item content suggested that both factors 1 and 2 are a mixture of cognitive and functional symptoms with factors 3 and 4 being primarily behavioral symptoms.

For the informant-report MANS, the 4 factor structure explained $80.3 \%$ of the variance. Factor 1 explained $63.1 \%$ of the variance (eigenvalue $=39.6$ ), factor 2 explained an additional $6.7 \%$ of the variance (eigenvalue = 4.21 ), factor 3 explained an additional 5.6\% of the variance (eigenvalue $=3.53$ ) and factor 4 explained $4.9 \%$ of the variance (eigenvalue $=3.05$ ). A review of item content suggested that factor 1 is primarily cognitive symptoms, factor 2 is primarily behavioral symptoms, factor 3 is primarily functional symptoms and factor 4 is primarily language symptoms.

\section{MANS Characteristics}

Table 3 provides detailed information on the performance of the participants on the MANS self-report version for the sample divided by unimpaired or impaired cognitive status. Table 3 also provides preliminary information on 2 diagnostic subgroups, $\mathrm{AD}(\mathrm{n}=17)$ and $\mathrm{MCI}$ $(\mathrm{n}=15)$. We caution that these preliminary subgroup scores should not be utilized as normative data, due to the small sample size and preliminary status of our work on the MANS. Table 3 provides information for both the overall MANS as well as the factors identified via factor analysis. $p<0.05$ was considered statistically significant. 
Table 1. Self-report MANS factor analysis standardized regression coefficients

In the past year have you noticed a change in daily habits? If yes, does that include:

Change in driving habits

Problems speaking or understanding spoken language

Problems with the ability to write

Getting lost

Managing money, paying bills or balancing the checkbook

Managing stocks, investments or assembling tax records

Handling money

Shopping

Problems with telephone solicitation or telephone marketing

Entering sweepstakes or contests

Reading, remembering or understanding what you read

Change in ability to spell

Change in patterns of watching television

Changes in driving patterns or abilities

Household maintenance or cleaning

Making unusual or impaired judgments

Change in personality or usual patterns of behavior

Problems with learning new things

Talking and thinking more about the past

Difficulty remembering recent events

Difficulty remembering names of familiar people

Losing or misplacing things

Difficulty remembering things or events from long ago

Difficulty with remembering planned activities or missing appointments

Keeping events or tasks in the right order

Becoming lost or asking directions in a place that should be familiar

Becoming mixed up about the time, day, date or year

Knowing where you are

Using the right word when speaking

Pronouncing words in the wrong way

Using words that do not exist or talking in incoherent or jumbled manner

Having difficulty naming objects

Losing a train of thought and not finishing sentences

Talking more than usual

Talking less than usual

Becoming nervous or anxious when talking to people

Giving unusually short answers to questions

Avoiding starting conversations

Recognizing familiar objects

Locating items in front of you

Problems using familiar objects such as programming the VCR, using the TV remote or the microwave

Has your personality changed in the past year? If yes, does that include:

Did you interpret things very literally, even when it might be inappropriate to do so

Did you seem to obsess on something (say or do something over and over that made you feel you were having problems thinking about something else)

Are there times when you behave out of character

Have you been less interested in usual activities, work, family or hobbies

Have you been more self-centered or selfish

Do you seem to act more impulsively, acting without appearing to consider the consequences

Have you become rude or insensitive to others

Making unusually large plans or purchases or making purchases that seem odd or irrational in their size or timing

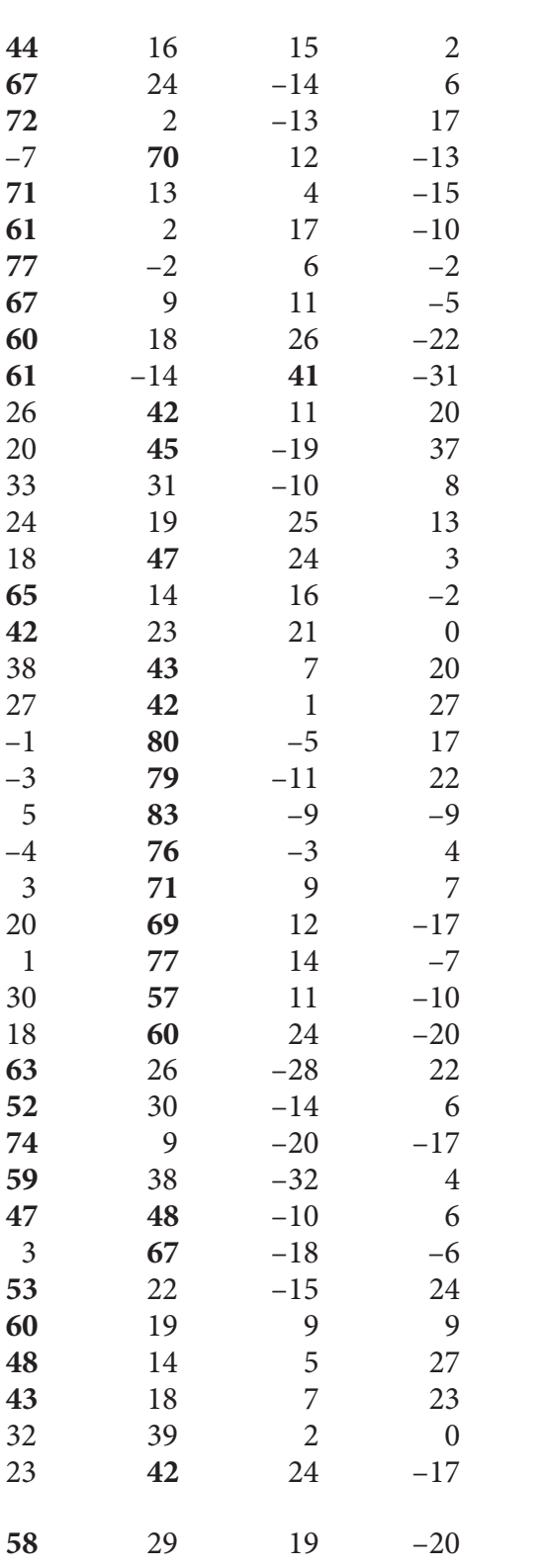

$\begin{array}{rrrr}11 & 16 & \mathbf{3 9} & 17 \\ \mathbf{4 1} & 18 & 8 & 12 \\ 37 & 20 & 20 & 11 \\ 35 & 25 & 19 & 24 \\ 35 & 10 & \mathbf{4 3} & -20 \\ 35 & -7 & \mathbf{4 6} & 25 \\ -16 & 4 & \mathbf{4 9} & \mathbf{4 5} \\ \mathbf{4 4} & -21 & \mathbf{5 7} & -19\end{array}$


Table 1 (continued)

\begin{tabular}{|c|c|c|c|c|}
\hline Item & Factor 1 & Factor 2 & Factor 3 & Factor 4 \\
\hline Obsessing on a particular idea or activity & 26 & 33 & 27 & -19 \\
\hline Behavioral incidents that seem bizarre, risky or dangerous & 45 & -46 & 23 & 43 \\
\hline Have you become less modest about displaying your body & 18 & 15 & 26 & 37 \\
\hline Laughing at unsuitable times & -10 & 26 & 18 & 15 \\
\hline Making jokes or comments that are considered to be in poor taste & -21 & 1 & 55 & 56 \\
\hline Display embarrassing behavior in public & -5 & -6 & 56 & 27 \\
\hline A change in your sexual desire or libido & -17 & 32 & 54 & 15 \\
\hline Talking to strangers as if familiar & -2 & 49 & 5 & 4 \\
\hline Using uncharacteristically coarse or profane language (cursing) & -9 & 14 & 16 & 40 \\
\hline Become less attentive to personal appearance and hygiene & 19 & 30 & 17 & -5 \\
\hline Become more aggressive or threatening & -9 & -3 & 68 & 15 \\
\hline Become more passive or less threatening & 38 & -16 & 28 & 27 \\
\hline Become more stubborn or set in your ways & 2 & 33 & 47 & 26 \\
\hline Developed repetitive activities or habits you perform over and over & 14 & 3 & 58 & 9 \\
\hline Become more distractible or have trouble concentrating & 17 & 29 & 38 & 27 \\
\hline Become restless or physically overactive & 8 & -1 & 69 & -1 \\
\hline Do you react more frequently to problems by becoming unusually upset or distressed & 3 & 47 & 46 & -4 \\
\hline Unusually anxious & -2 & 30 & 45 & 28 \\
\hline Lose confidence & 36 & 29 & 19 & 16 \\
\hline Do you seem to cry more easily & 13 & 15 & 9 & 44 \\
\hline Hoard objects or hide things & 1 & -1 & 59 & 14 \\
\hline Complain without justification that things have been stolen & -12 & -3 & 80 & -28 \\
\hline See or hear things not experience by others & 6 & 36 & 19 & -6 \\
\hline $\begin{array}{l}\text { Complain of family members or neighbors with 'unseemly' conduct such as criminal } \\
\text { activity, drugs, taking things or having an illicit relationship }\end{array}$ & -26 & 27 & 30 & -2 \\
\hline Do you become tearful or depressed & -1 & 28 & 28 & 46 \\
\hline Do you seem happier or more enthusiastic than usual & 22 & 1 & 20 & 7 \\
\hline Are you more irritable or impatient & -1 & 30 & 44 & 24 \\
\hline Do your moods change rapidly during the day & 15 & 14 & 33 & 41 \\
\hline Do you seem emotionally blunted, not responding to good or bad news in a predictable way & 33 & -30 & 51 & 30 \\
\hline Do you seem to over-react & -17 & 27 & 51 & 12 \\
\hline Do you seem apathetic at times & 37 & -17 & 12 & 52 \\
\hline
\end{tabular}

In the past year, have your movements changed? If yes, does that include:

Have you noticed twitching or jerking

Have you noticed a tremor

Have you noticed any abnormal movements

Do you seem stiff or rigid

Do your movements seem slowed

Do you have a problem walking

Eigenvalues

\begin{tabular}{rrrr}
13 & -2 & -5 & $\mathbf{6 1}$ \\
-5 & 0 & -6 & $\mathbf{6 9}$ \\
-5 & -7 & 1 & $\mathbf{7 0}$ \\
$\mathbf{6 4}$ & -14 & -11 & 15 \\
$\mathbf{6 5}$ & -21 & 0 & 31 \\
$\mathbf{6 0}$ & -20 & -6 & 28 \\
\hline 31.1 & 4.96 & 3.86 & 3.51
\end{tabular}

Values are multiplied by 100 and rounded to nearest integer. Values greater than 40 are in bold. The complete questionnaire with instructions, stem questions and formatting is available from the authors.

All unimpaired participants completed the self-report MANS, but there were 3 cognitively impaired participants who did not complete the self-report MANS. Figure 1 shows the distribution of scores on the MANS selfreport version divided into cognitive status groups. There it can be seen that the unimpaired group data have a skewed distribution, as appropriate, while the partici- pants with impaired cognitive status display a more normalized distribution of scores across the spectrum of possible scores. Statistical comparison (Mann Whitney U) of participants with unimpaired cognition and participants with impaired cognition reveals a significant difference between groups on the self-report MANS, as expected $(z=-6.45, p<0.001)$. Cohen's d effect size for 
Table 2. Informant-report MANS factor analysis standardized regression coefficients

Item

Factor 1 Factor 2 Factor 3 Factor 4

In the past year have you noticed a change in daily habits? If yes, does that include:

Change in driving habits

Problems speaking or understanding spoken language

Problems with the ability to write

Getting lost

Managing money, paying bills or balancing the checkbook

Managing stocks, investments or assembling tax records

Handling money

Shopping

Problems with telephone solicitation or telephone marketing

Entering sweepstakes or contests

Reading, remembering or understanding what you read

Change in ability to spell

Change in patterns of watching television

Changes in driving patterns or abilities

Household maintenance or cleaning

Making unusual or impaired judgments

Change in personality or usual patterns of behavior

Problems with learning new things

Talking and thinking more about the past

Difficulty remembering recent events

Difficulty remembering names of familiar people

Losing or misplacing things

Difficulty remembering things or events from long ago

Difficulty with remembering planned activities or missing appointments

Keeping events or tasks in the right order

Becoming lost or asking directions in a place that should be familiar

Becoming mixed up about the time, day, date or year

Knowing where you are

Using the right word when speaking

Pronouncing words in the wrong way

Using words that do not exist or talking in incoherent or jumbled manner

Having difficulty naming objects

Losing a train of thought and not finishing sentences

Talking more than usual

Talking less than usual

Becoming nervous or anxious when talking to people

Giving unusually short answers to questions

Avoiding starting conversations

Recognizing familiar objects

Locating items in front of you

Problems using familiar objects such as programming the VCR, using the TV remote or the microwave

Has your personality changed in the past year? If yes, does that include:

Did you interpret things very literally, even when it might be inappropriate to do so

Did you seem to obsess on something (say or do something over and over that made you feel you were having problems thinking about something else)

Are there times when you behave out of character

Have you been less interested in usual activities, work, family or hobbies

Have you been more self-centered or selfish

Do you seem to act more impulsively, acting without appearing to consider the consequences

Have you become rude or insensitive to others

Making unusually large plans or purchases or making purchases that seem odd or irrational in their size or timing

12

61

49

$-6$

21

14

24

13

33

27

11

37

21

10

11

33

11

21

23

$-11$

9

$-12$

22

$-16$

15

$-10$

$-2$

$-3$

75

87

76

64

58

12

34

51

53

29

18

25

$57-2$

69

50

$-4$

14

26

50

$-27$

36

$\begin{array}{llll}56 & 28 & 1 & 9\end{array}$

$\begin{array}{llll}26 & 59 & -17 & 22\end{array}$

$11 \quad 32$

$31 \quad 56$

$61-4$

$\begin{array}{rrrr}9 & 57 & 23 & 17\end{array}$

$\begin{array}{llll}-8 & 82 & 16 & -12\end{array}$

$\begin{array}{llll}-5 & 50 & -3 & 26\end{array}$ 
Table 2 (continued)

\begin{tabular}{|c|c|c|c|c|}
\hline Item & Factor 1 & Factor 2 & Factor 3 & Factor 4 \\
\hline Obsessing on a particular idea or activity & 9 & 52 & 7 & 27 \\
\hline Behavioral incidents that seem bizarre, risky or dangerous & -33 & 37 & 36 & 40 \\
\hline Have you become less modest about displaying your body & 18 & 35 & 20 & -6 \\
\hline Laughing at unsuitable times & 29 & 61 & -8 & -9 \\
\hline Making jokes or comments that are considered to be in poor taste & -14 & 81 & -7 & 2 \\
\hline Display embarrassing behavior in public & 3 & 73 & 3 & -16 \\
\hline A change in your sexual desire or libido & 7 & 54 & 11 & -6 \\
\hline Talking to strangers as if familiar & 43 & 46 & -14 & 10 \\
\hline Using uncharacteristically coarse or profane language (cursing) & -9 & 68 & 11 & -1 \\
\hline Become less attentive to personal appearance and hygiene & 27 & 44 & 41 & -32 \\
\hline Become more aggressive or threatening & -41 & 46 & 32 & 44 \\
\hline Become more passive or less threatening & 18 & 4 & 73 & -33 \\
\hline Become more stubborn or set in your ways & 22 & 54 & 8 & 17 \\
\hline Developed repetitive activities or habits you perform over and over & 30 & 36 & 13 & 16 \\
\hline Become more distractible or have trouble concentrating & 36 & 19 & 42 & 7 \\
\hline Become restless or physically overactive & -7 & 18 & 23 & 44 \\
\hline Do you react more frequently to problems by becoming unusually upset or distressed & 29 & 44 & 10 & 20 \\
\hline Unusually anxious & 34 & 25 & 18 & 20 \\
\hline Lose confidence & 26 & 17 & 30 & 31 \\
\hline Do you seem to cry more easily & 22 & 27 & 23 & 0 \\
\hline Hoard objects or hide things & 28 & 46 & -8 & 7 \\
\hline Complain without justification that things have been stolen & 25 & 34 & -15 & 17 \\
\hline See or hear things not experience by others & 27 & 20 & 3 & 8 \\
\hline $\begin{array}{l}\text { Complain of family members or neighbors with 'unseemly' conduct such as criminal } \\
\text { activity, drugs, taking things or having an illicit relationship }\end{array}$ & 16 & 46 & -15 & 4 \\
\hline Do you become tearful or depressed & 17 & 29 & 48 & -10 \\
\hline Do you seem happier or more enthusiastic than usual & 25 & 12 & -1 & 16 \\
\hline Are you more irritable or impatient & 17 & 63 & 5 & 10 \\
\hline Do your moods change rapidly during the day & 15 & 51 & -6 & 42 \\
\hline Do you seem emotionally blunted, not responding to good or bad news in a predictable way & -34 & 44 & 51 & 23 \\
\hline Do you seem to over-react & 12 & 68 & -13 & 19 \\
\hline Do you seem apathetic at times & 12 & 26 & 61 & -6 \\
\hline
\end{tabular}

In the past year, have your movements changed? If yes, does that include:

Have you noticed twitching or jerking

Have you noticed a tremor

Have you noticed any abnormal movements

Do you seem stiff or rigid

Do your movements seem slowed

Do you have a problem walking

Eigenvalues

\begin{tabular}{rrrr}
-1 & 18 & 28 & 15 \\
-15 & -7 & $\mathbf{4 1}$ & 3 \\
1 & $\mathbf{4 4}$ & 30 & -3 \\
9 & 3 & $\mathbf{5 1}$ & -5 \\
7 & 3 & $\mathbf{8 0}$ & -18 \\
3 & -8 & $\mathbf{7 3}$ & -6 \\
\hline 39.6 & 4.21 & 3.53 & 3.05
\end{tabular}

Values are multiplied by 100 and rounded to nearest integer. Values greater than 40 are in bold. The complete questionnaire with instructions, stem questions and formatting is available from the authors.

the difference between groups on the self-report MANS is -1.29 . Comparison of the subgroups of MCI patients and $\mathrm{AD}$ patients shows a significant difference with $\mathrm{MCI}$ groups having lower (and therefore better) scores when compared to the AD group $(\mathrm{z}=-2.51, \mathrm{p}=0.01)$. Cohen's $\mathrm{d}$ effect size for the difference between the MCI and $\mathrm{AD}$ subgroups on the self-report MANS is -0.91 .
Table 3 also provides detailed information on the informant-report version of the MANS by cognitive status. Again, information is provided for both the overall MANS as well as the factors identified by factor analysis. A total of 7 participants did not have an informant complete the MANS (5 unimpaired, 2 impaired). Figure 2 is a graphic representation of the distributions. Similar to 
Table 3. Descriptive statistics on the MANS by total sample and diagnostic group

\begin{tabular}{|c|c|c|c|c|}
\hline & Unimpaired & Impaired & MCI & $\mathrm{AD}$ \\
\hline Self-report & $(\mathrm{n}=65)$ & $(\mathrm{n}=57)$ & $(\mathrm{n}=15)$ & $(\mathrm{n}=16)$ \\
\hline Mean raw score $\pm S D$ & $19.3 \pm 30.4$ & $75.9 \pm 59.0$ & $51.6 \pm 41.5$ & $97.7 \pm 52.4$ \\
\hline Median & 4 & 63 & 57 & 89 \\
\hline Minimum & 0 & 0 & 0 & 6 \\
\hline Maximum & 143 & 232 & 159 & 173 \\
\hline \multicolumn{5}{|c|}{ Factor, mean raw $\pm \mathrm{SD}$ (median) } \\
\hline 1 & $4.8 \pm 8.8(0)$ & $25.7 \pm 23.5(18)$ & $12.3 \pm 9.5(12)$ & $30.1 \pm 19.1(24.5)$ \\
\hline 2 & $7.1 \pm 11.2(0)$ & $24.5 \pm 18.6(23)$ & $19.5 \pm 15.5(20)$ & $35.1 \pm 16.6(33.5)$ \\
\hline 3 & $3.0 \pm 5.9(0)$ & $11.6 \pm 13.3(8)$ & $9.8 \pm 11.2(8)$ & $14.3 \pm 16.4(9.5)$ \\
\hline 4 & $2.1 \pm 4.1(0)$ & $5.3 \pm 7.6(2)$ & $4.7 \pm 6.7(2)$ & $5.7 \pm 9.8(2)$ \\
\hline Informant-report & $(\mathrm{n}=60)$ & $(\mathrm{n}=58)$ & $(\mathrm{n}=15)$ & $(\mathrm{n}=17)$ \\
\hline Mean raw score $\pm \mathrm{SD}$ & $13.7 \pm 27.3$ & $106.3 \pm 72.8$ & $62.4 \pm 56.3$ & $149.7 \pm 60.9$ \\
\hline Median & 0 & 103 & 49 & 154 \\
\hline Minimum & 0 & 0 & 0 & 50 \\
\hline Maximum & 131 & 315 & 199 & 315 \\
\hline \multicolumn{5}{|c|}{ Factor, mean raw $\pm \mathrm{SD}$ (median) } \\
\hline 1 & $4.1 \pm 8.9(0)$ & $36.2 \pm 23.8(31.5)$ & $25.5 \pm 18.4(24)$ & $54.2 \pm 17.2(58)$ \\
\hline 2 & $4.1 \pm 8.8(0)$ & $20.4 \pm 21.0(15)$ & $12.5 \pm 20.9(5)$ & $31.0 \pm 21.8(28)$ \\
\hline 3 & $3.1 \pm 6.7(0)$ & $32.9 \pm 22.5(35)$ & $18.4 \pm 16.7(15)$ & $41.5 \pm 15.1(44)$ \\
\hline 4 & $2.1 \pm 5.1(0)$ & $14.5 \pm 12.7(12)$ & $5.6 \pm 6.7(4)$ & $17.8 \pm 12.7(15)$ \\
\hline
\end{tabular}

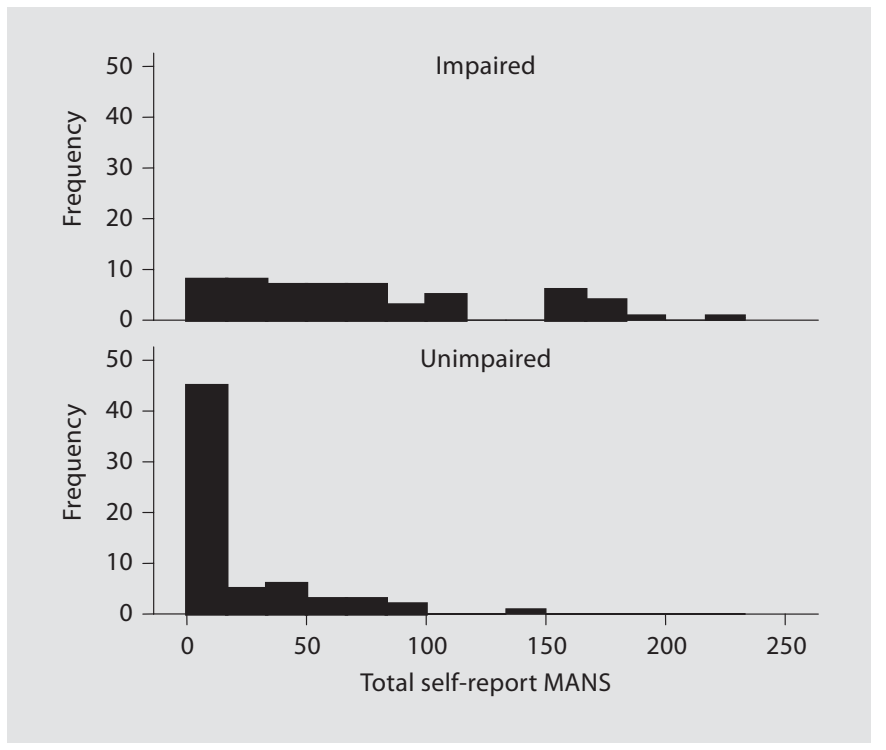

Fig. 1. Distribution of self-report MANS scores by cognitive status.

the self-report version of the MANS, the score distribution is very negatively skewed for the unimpaired subgroup with a large portion of the informants rating the participant as having no change in the past year. Statistical comparison (Mann Whitney U) of informant-report

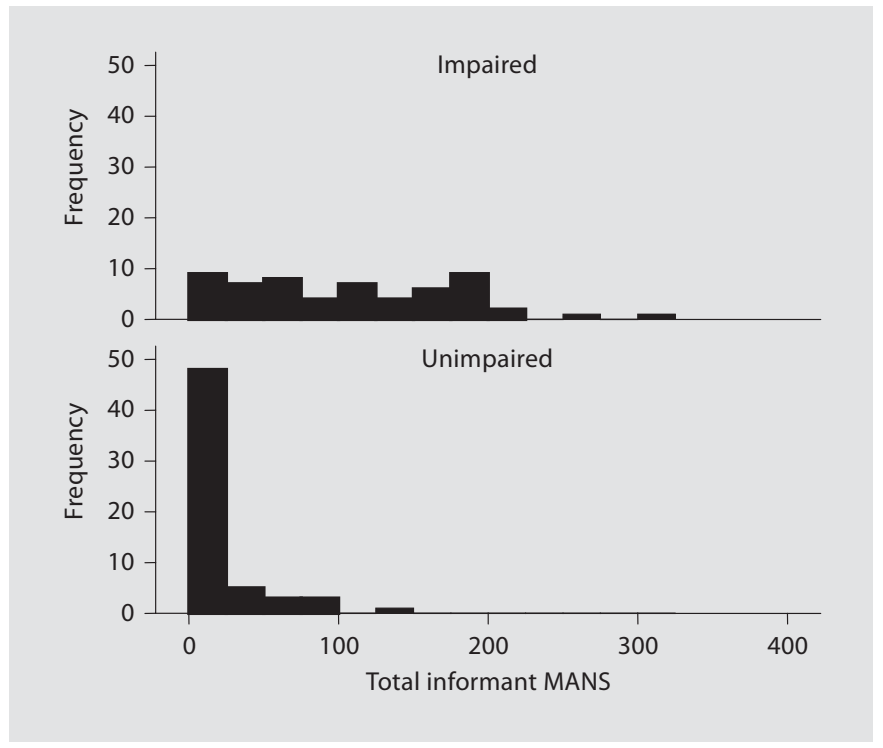

Fig. 2. Distribution of informant-report MANS scores by cognitive status.

MANS of unimpaired versus impaired participants on the MANS shows a significant difference between groups, as expected ( $z=-7.87, p<0.001)$. Cohen's d effect size for the difference between groups on the informant-report MANS is -1.86 . Comparison of the subgroups of MCI 
patients and $\mathrm{AD}$ patients on the informant-report MANS shows a significant difference, with the MCI groups having lower (and therefore better) scores when compared to the $\mathrm{AD}$ group $(\mathrm{z}=-3.49, \mathrm{p}<0.0001)$. Cohen's d effect size for the difference between $\mathrm{MCI}$ and $\mathrm{AD}$ on the informant report MANS is -1.49 .

Comparison (Wilcoxon Signed Ranks Test) between the self-report and informant-report MANS scores shows that across the entire sample there is no significant difference between self-report and informant-report MANS scores $(\mathrm{z}=-1.28, \mathrm{p}=0.20)$. Similarly, correlational analysis shows a large correlation between self-report MANS and informant-report MANS $(\mathrm{r}=0.64, \mathrm{p}<0.001)$. However, when broken into diagnostic groups, those with unimpaired cognition rated themselves as having more symptoms than did their informants $(\mathrm{z}=-2.22, \mathrm{p}=0.03)$, but those with impaired cognition rated themselves as having fewer symptoms than did their informants $(\mathrm{z}=$ $-2.64, \mathrm{p}=0.008)$. Despite these differences, the relationship between self-report and informant-report MANS scores was moderate to large for both groups (unimpaired cognition $\mathrm{r}=0.66, \mathrm{p}<0.001$; impaired cognition $\mathrm{r}=0.42$, $\mathrm{p}=0.001$ ).

Table 4 provides age distribution and gender distribution by diagnostic group. The unimpaired cognition group is slightly younger than the impaired cognition group ( $\mathrm{p}=0.04)$, and the unimpaired cognition group has more women $(65 \%)$ than the impaired cognition group $(43 \%, p=0.02)$. Further analysis of the relationship between age and MANS scores shows a small but significant positive correlation between age and self-report MANS $(\mathrm{r}=0.14, \mathrm{p}=0.03)$ and a small correlation between age and informant-report MANS $(r=0.14, p=0.04)$, suggesting that older participants have more symptoms. Further analysis of the relationship between gender and MANS reveals no difference between MANS self-report scores for women $(M=41.0, S D=51.7$, median $=16.5)$ and men $(\mathrm{M}=51, \mathrm{SD}=56.3$, median $=33.5)$ across the sample as a whole $(\mathrm{z}=-1.34, \mathrm{p}=0.18)$. However, across the sample as a whole, male participants $(\mathrm{M}=71.8, \mathrm{SD}=70.2$, median $=53)$ tend to be rated higher (i.e. more impaired) than female participants $(\mathrm{M}=48.6, \mathrm{SD}=71.5$, median $=$ $8.5)$ by their informants $(z=-2.67, p<0.01)$. Similarly, across the sample as a whole, male participants are more impaired than female participants on the DRS-2 $(\mathrm{z}=$ $-2.107, p=0.04)$ and there is a similar trend on the MMSE $(z=-1.90, p=0.06)$. Thus, it is possible that the men in this sample were statistically more impaired than the women in this sample, and the informant-report MANS differences reflect that.
Table 4. Sample characteristics by diagnostic group

\begin{tabular}{|c|c|c|c|c|c|}
\hline \multicolumn{2}{|l|}{ Variable } & Unimpaired & Impaired & $\mathrm{p}$ & d \\
\hline \multirow{2}{*}{\multicolumn{2}{|c|}{$\begin{array}{l}\text { Gender (female), \% } \\
\text { n }\end{array}$}} & 65 & 43 & 0.02 & $\mathrm{~N} / \mathrm{A}$ \\
\hline & & 65 & 60 & & \\
\hline \multirow[t]{4}{*}{ Age } & Mean $\pm S D$ & $69.2 \pm 6.9$ & $71.9 \pm 7.7$ & & \\
\hline & Median & 69.0 & 73 & 0.04 & -0.37 \\
\hline & Range & 50-84 & $52-85$ & & \\
\hline & $\mathrm{n}$ & 65 & 60 & & \\
\hline \multirow[t]{4}{*}{ Ham-D } & Mean \pm SD & $1.7 \pm 2.1$ & $2.8 \pm 3.4$ & 0.05 & -0.41 \\
\hline & Median & 1.0 & 2.0 & & \\
\hline & Range & $0-7$ & $0-17$ & & \\
\hline & $\mathrm{n}$ & 65 & 54 & & \\
\hline \multirow[t]{4}{*}{ GDS } & Mean $\pm S D$ & $2.9 \pm 3.0$ & $7.6 \pm 5.3$ & $<0.001$ & -1.16 \\
\hline & Median & 2.0 & 7.5 & & \\
\hline & Range & $0-13$ & $0-21$ & & \\
\hline & $\mathrm{n}$ & 65 & 54 & & \\
\hline \multirow[t]{4}{*}{ FAQ } & Mean $\pm S D$ & $0.03 \pm 0.17$ & $11.4 \pm 9.3$ & $<0.001$ & -2.67 \\
\hline & Median & 0 & 10 & & \\
\hline & Range & $0-1$ & $0-30$ & & \\
\hline & $\mathrm{n}$ & 65 & 53 & & \\
\hline NPI & Mean \pm SD & $1.03 \pm 1.9$ & $4.4 \pm 5.0$ & $<0.001$ & -1.03 \\
\hline \multirow[t]{3}{*}{ Severity } & Median & 0 & 3.0 & & \\
\hline & Range & $0-8$ & $0-20$ & & \\
\hline & $\mathrm{n}$ & 63 & 51 & & \\
\hline \multirow[t]{4}{*}{ UPDRS } & Mean $\pm S D$ & $0.14 \pm 0.8$ & $3.9 \pm 7.9$ & 0.001 & -0.92 \\
\hline & Median & 0 & 0 & & \\
\hline & Range & $0-5$ & $0-31$ & & \\
\hline & $\mathrm{n}$ & 65 & 56 & & \\
\hline \multirow[t]{4}{*}{ MMSE } & Mean \pm SD & $29.6 \pm 0.63$ & $23.3 \pm 7.1$ & $<0.001$ & 1.68 \\
\hline & Median & 30 & 26.5 & & \\
\hline & Range & $27-30$ & $2-30$ & & \\
\hline & $\mathrm{n}$ & 64 & 60 & & \\
\hline \multirow[t]{4}{*}{ DRS-2 } & Mean \pm SD & $139.9 \pm 3.3$ & $113.8 \pm 26.1$ & $<0.001$ & 1.85 \\
\hline & Median & 141 & 124 & & \\
\hline & Range & $127-144$ & $23-140$ & & \\
\hline & $\mathrm{n}$ & 65 & 59 & & \\
\hline \multirow[t]{6}{*}{ CDR, \% } & 0 & 97 & 5 & $<0.001$ & N/A \\
\hline & 0.5 & 3 & 59 & & \\
\hline & 1.0 & 0 & 22 & & \\
\hline & 2.0 & 0 & 7 & & \\
\hline & 3.0 & 0 & 7 & & \\
\hline & $\mathrm{n}$ & 65 & 59 & & \\
\hline
\end{tabular}

Statistical comparisons include $\chi^{2}$ analyses for categorical data (gender, CDR), $t$ test with equal variance assumed (age), and t test with equal variances not assumed (Ham-D, GDS, FAQ, NPI Severity, UPDRS, MMSE, DRS). $\mathrm{d}$ = Cohen's d effect size; Ham-D = Hamilton Depression Scale; GDS = Geriatric Depression Scale; FAS = Functional Assessment Scale $;$ NPI = Neuropsychiatric Inventory; UPDRS = Unified Parkinson's Disease Rating Scale; MMSE = Mini Mental State Exam; DRS-2 = Dementia Rating Scale-2nd Edition; CDR = Clinical Dementia Rating scale. 


\section{Construct Validity}

Table 4 provides more detailed information about the performances of each diagnostic group on the additional measures collected as part of the longitudinal project. As can be seen, the group with unimpaired cognition performed statistically better on all measures than the group with impaired cognition, as expected. Effect sizes and raw data are also provided where appropriate in order to provide information on clinical relevance in addition to statistical significance. As shown, the majority of the effect sizes were quite large, suggesting clinically meaningful difference as well as statistical difference.

Table 5 outlines the correlational relationships between the self-report and informant-report MANS and the related construct measures outlined in the methods section. Scatterplots of these distributions suggested 1 likely outlier on the basis of an unusually high Ham-D score and 1 likely outlier on the basis of an unusually low DRS-2 score and those were removed from this analysis. As can be seen, all correlations between the self-report MANS and the related construct measures were significant in the expected strengths and directions. Most correlations are moderate to large with 0.54 between the $\mathrm{Ge}$ riatric Depression Scale and the MANS and between the FAQ and the MANS, -0.57 between the DRS- 2 total score and the MANS and 0.64 between the CDR and the MANS. The smallest correlation is 0.26 between the UPDRS and the MANS. Similarly, the correlations between the informant-report MANS and the related construct measures were significant in the expected strengths and directions. There are several quite large correlations, with -0.63 between the DRS- 2 and the informant MANS, 0.80 between the CDR and the MANS and 0.87 between the FAQ and the MANS. Again, the smallest correlation is 0.26 between the UPDRS and the informant MANS.

\section{Discussion}

The primary aim of this study was to provide initial psychometric and construct validation information for the MANS, new questionnaires pairing informant- and self-report for the assessment of cognitive, functional, behavioral and motor symptoms that may be related to various neurodegenerative etiologies. Extant tools attempt to quantify subjective observation, but several properties of the MANS render it unique. Like the CDR, the MANS pairs informant with self observations, but unlike the $\mathrm{CDR}$, the MANS does not require administration by an examiner. The MANS can be completed within $10 \mathrm{~min}$ if
Table 5. Correlations between MANS and related construct measures

\begin{tabular}{lcrlr}
\hline Measure & $\begin{array}{l}\text { Self- } \\
\text { report }\end{array}$ & $p$ & $\begin{array}{l}\text { Informant- } \\
\text { report }\end{array}$ & $p$ \\
\hline Ham-D & $0.31^{1}$ & 0.001 & $0.35^{1}$ & $<0.001$ \\
GDS & 0.54 & $<0.001$ & 0.37 & $<0.001$ \\
FAQ & 0.54 & $<0.001$ & 0.87 & $<0.001$ \\
NPI Severity & 0.44 & $<0.001$ & 0.72 & $<0.001$ \\
UPDRS & 0.26 & 0.004 & 0.26 & 0.001 \\
MMSE & -0.55 & $<0.001$ & -0.65 & $<0.001$ \\
CDR & 0.64 & $<0.001$ & 0.80 & $<0.001$ \\
DRS-2 & $-0.57^{1}$ & $<0.001$ & $-0.63^{1}$ & $<0.001$ \\
\hline
\end{tabular}

Correlations with Pearson's $r$ for all except the CDR, which was evaluated with Spearman's rho. See text or table 4 for explanation of abbreviations.

${ }^{1}$ Outlier removed.

there is impairment, and within a minute if not. The MANS generally correlates well with other commonly employed construct measures (table 5) while providing greater breadth of symptom surveillance and a convenient self-administered format.

The results of this study provide initial evidence suggesting that the MANS is a psychometrically sound and construct valid measure to aid in the detection of symptoms related to neurodegenerative illness. The summary statistics show that the MANS has adequate variability to be meaningful. That is, participants' scores were varied along the entire spectrum of possible scores on the scales, providing clinically meaningful data. Individuals with normal cognition scored better on both self-report and informant-report MANS when compared with those with impaired cognition. Preliminary subgroup analysis showed that MCI patients scored better on both versions of the MANS when compared to patients with $\mathrm{AD}$. Internal consistencies of the MANS self-report and informant versions are excellent.

Four factors were found embedded within our conceptually conceived 3 categories of change (cognitive, behavioral and movement). This supports the multi-dimensional nature of the MANS, and while the items showed great overlap between informant and self versions, the items did not always fall into the same factor, underscoring the importance of all 4 factors for comparison between informant and self. For example, of 6 motor questions (all of which might seem straightforward), 4 were in factor 3 for the informant, but all were in either factor 1 or factor 4 for the self version.
Locke/Dassel/Hall/Baxter/Woodruff/ Hoffman Snyder/Miller/Caselli 
Some items did not load significantly on any of the 4 factors. Upon review of those items, many involved symptoms that would be expected to have a relatively low base rate in both normal and impaired populations. Examples include, 'Has your loved one become less modest about displaying his/her body?' and 'Have you seen or heard things not experienced by others?'. These symptoms, when present, are usually highly clinically relevant and therefore are maintained on the MANS at this point. A necessary next step would be analysis of the structure of the MANS within various diagnostic groups with attention to these rare but relevant symptoms and their diagnostic utility without changing the accuracy of distinguishing other subtle signs of dementia through the other MANS items. We do not have the sample size to do this at this point.

There are several limitations of this study. First, we have not yet assessed longitudinal stability in those individuals who appear to be clinically stable on other measures, but future analysis is planned for our participants with multiple epochs of data. Second, we have not yet addressed test-retest reliability for the MANS or inter-rater reliability when different informants are compared. Third, the majority of impaired participants in this sample had CDR scores of 0.5 or 1 . While our primary intent has been to develop a measure for early detection of neurodegenerative symptoms, more experience with later stages of impairment is still needed. Finally, this study consists primarily of patients with AD in comparison to participants with normal cognition. Future studies including more rarely occurring neurodegenerative disor- ders are planned to allow us to determine how well this measure captures symptoms present in a broader group of dementias.

In summary, this work provides preliminary validation information of the MANS, a measure of cognitive, personality, functional and motor symptoms occurring in patients developing dementia. The MANS holds promise as a tool that combines the strengths of many other measures in a brief and easy to administer form to help with both diagnosis of patients in the early stages of dementia as well as providing a more broad assessment of the common symptoms that require monitoring and treatment in the typical clinical setting. Additional validation information is required before the MANS can be used clinically, but these preliminary results support further work with the MANS in research settings with the goal of establishing that clinical utility.

Readers interested in obtaining a copy of the MANS should contact Drs. Locke or Caselli at the Mayo Clinic in Scottsdale, Ariz., USA. Contact details are on the first page of this report.

\section{Acknowledgments}

The authors would like to thank study coordinators Sandra Yee-Benedetto and Bruce Henslin for their work on the ADC project and the APOE cohort, and Amylou Dueck, PhD, for statistical review. Thank you also to Kirk Wilhelmsen, $\mathrm{MD}, \mathrm{PhD}$, and the FTD working group of the California State Alzheimer's Centers members for allowing us to alter their original telephone screening questionnaire.

\section{References}

1 Alzheimer's Association: 2008 Alzheimer's disease facts and figures. Alzheimers Dement 2008;4:110-133.

-2 Petersen RC, Smith GE, Waring SC, Ivnik RJ, Tangalos EG, Kokmen E: Mild cognitive impairment: clinical characterization and outcome. Arch Neurol 1999;56:303-308.

-3 Petersen RC, Stevens JC, Ganguli M, Tangalos EG, Cummings JL, DeKosky ST: Practice parameter: early detection of dementia: mild cognitive impairment (an evidence-based review). Report of the Quality Standards Subcommittee of the American Academy of Neurology. Neurology 2001;56:1133-1142.

4 American Psychiatric Association: Diagnostic and Statistical Manual of Mental Disorders, ed 4. Washington, American Psychiatric Association, 1994.
-5 Saykin AJ, Wishart HA, Rabin LA, Santulli RB, Flashman LA, West JD, et al: Older adults with cognitive complaints show brain atrophy similar to that of amnestic MCI. Neurology 2006;67:834-842.

-6 Isella V, Villa L, Russo A, Regazzoni R, Ferrarese C, Appollonio IM: Discriminative and predictive power of an informant report in mild cognitive impairment. J Neurol Neurosurg Psychiatry 2006;77:166-171.

7 Ready RE, Ott BR, Grace J: Patient versus informant perspectives of Quality of Life in Mild Cognitive Impairment and Alzheimer's disease. Int J Geriatr Psychiatry 2004;19: 256-265. $\checkmark 8$ Albert SM, Michaels K, Padilla M, Pelton G, Bell K, Marder K, et al: Functional significance of mild cognitive impairment in elderly patients without a dementia diagnosis. Am J Geriatr Psychiatry 1999;7:213-220.

-9 Applegate WB, Blass JP, Williams TF: Instruments for the functional assessment of older patients. N Engl J Med 1990;322:12071214 .

10 Cummings JL, Mega M, Gray K, RosenbergThompson S, Carusi DA, Gornbein J: The Neuropsychiatric Inventory: comprehensive assessment of psychopathology in dementia. Neurology 2004;44:2308-2314.

11 Lawton MP, Brody EM: Assessment of older people: self maintaining and instrumental activities of daily living. Gerontologist 1969; 9:179-186. 
12 Gilewski MJ, Zelinski EM: Memory Functioning Questionnaire (MFQ). Psychopharmacol Bull 1988;24:665-670.

13 Morris JC: The Clinical Dementia Rating (CDR): current version and scoring rules. Neurology 1993;43:2412-2414.

-14 Reisberg B, Ferris SH, de Leon MJ, Crook T: The Global Deterioration Scale for assessment of primary degenerative dementia. Am J Psychiatry 1982;139:1136-1139.

15 Feinstein AR, Josephy BR, Wells CK: Scientific and clinical problems in indexes of functional disability. Ann Intern Med 1986; 105:413-420.

16 Guccione AA: Functional assessment of the elderly; in White K (ed): Geriatric Physical Therapy, ed 2. St. Louis, Mosby, 2000, pp 123-133.

-17 Caselli RJ, Reiman EM, Osborne D, Hentz JG, Baxter LC, Hernandez JL, Alexander GG: Longitudinal changes in cognition and behavior in asymptomatic carriers of the APOE e4 allele. Neurology 2004;62:19901995.
18 Reiman EM, Caselli RJ, Yun LS, Chen K, Bandy D, Minoshima S, Thibodeau SN, Osborne D: Preclinical evidence of Alzheimer's disease in persons homozygous for the epsilon 4 allele for apolipoprotein E. N Engl J Med 1996;334:752-758.

19 Crum RM, Anthony JC, Bassett SS, Folstein MF: Population-based norms for the minimental state examination by age and education level. JAMA 1993;269:2386-2391.

20 Hamilton M: A rating scale for depression. J Neurol Neurosurg Psychiatry 1960;23:5662

21 Hedlund JL, Vieweg BW: The Hamilton Rating Scale for Depression: a comprehensive review. J Oper Psychiatry 1979;10:149-165.

22 American Psychiatric Association: Diagnostic and statistical manual of mental disorders, ed 3, revised. Washington, American Psychiatric Association, 1987.

23 Pfeffer RI, Kurosaki TT, Harrah CH, Chance JM, Filos S: Measurement of the functional activities in older adults in the community. J Geront 1982;37:323-329.

24 Crook T, Bartus RT, Ferris SH, Whitehouse P, Cohen GD, Gershon S: Age-associated memory impairment: proposed diagnostic criteria and measures of clinical change: report of a National Institute of Mental Health work group. Dev Neuropsychol 1986;2:261276.
25 McKhann G, Drachman D, Folstein M, Katzman R, Price D, Stadlan EM: Clinical diagnosis of Alzheimer's disease: report of the NINCDS-ADRDA Work Group under the auspices of Department of Health and Human Services Task Force on Alzheimer's Disease. Neurology 1984;34:939-944.

26 Yesavage JA, Brink TL, Rose TL, Lum O, Huang V, Adey MB, Leirer VO: Development and validation of a geriatric depression rating scale: a preliminary report. J Psychiatr Res 1983;17:37-49.

27 Jurica PJ, Leitten CL, Mattis S: Dementia Rating Scale-2. Lutz, Psychological Assessment Resources, 2001.

28 Fahn S, Elton RL; the UPDRS Development Committee: Unified Parkinson's Disease Rating Scale; in Fahn S, Marsden CD, Calne D, Goldstein M (eds): Recent Developments in Parkinson's Disease. Florham Park, Macmillan Healthcare Information, 1987, vol 2, pp 153-163.

29 Cohen J: Statistical Power Analysis for the Behavioral Sciences, ed 2. Hillsdale, Lawrence Erlbaum Associates, 1998. 